\title{
Le gisement Pléistocène moyen de Puig d'en Roca (Espagne)
}

\section{E. Carbonell, J. Collina-Girard, R. Mora, R. Sala, M. Guilbaud}

\section{Résumé}

RESUME La fouille d'un colluvion d'une haute terrasse du Ter (Gérone, Espagne) a fourni une industrie archaïque en quartz, probablement mindélienne ; l'étude typologique, originale, est basée sur l'analyse du débitage.

\section{Citer ce document / Cite this document :}

Carbonell E., Collina-Girard J., Mora R., Sala R., Guilbaud M. Le gisement Pléistocène moyen de Puig d'en Roca (Espagne). In: Bulletin de la Société préhistorique française, tome $85, n^{\circ} 7,1988$. pp. 204-209;

doi : $10.3406 /$ bspf. 1988.9344

http://www.persee.fr/doc/bspf_0249-7638_1988_num_85_7_9344

Document généré le 09/04/2016 


\title{
Le gisement Pléistocène moyen de Puig d'en Roca (Espagne)
}

\author{
par E. Carbonell, J. Collina-Girard, M. Guilbaud, R. Mora, R. Sala
}

\section{RÉSUMÉ}

La fouille d'un colluvion d'une haute terrasse du Ter (Gérone, Espagne) a fourni une industrie archaïque en quartz, probablement mindélienne; l'étude typologique, originale, est basée sur l'analyse du débitage.

\section{SITUATION}

Le gisement de Puig d'en Roca, d'âge Pléistocène moyen, se situe au nord-est de la péninsule ibérique tout près de Girona (Catalogne, Espagne) dans l'ensemble des terrasses quaternaires du Ter (fig. 1).

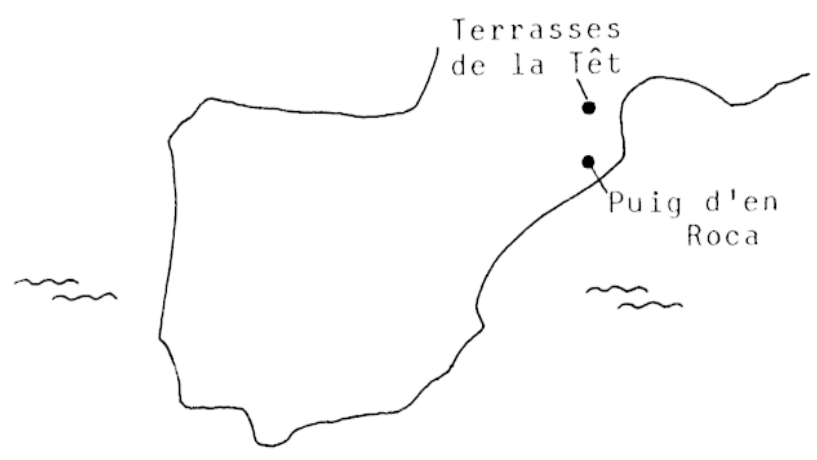

Fig. 1 - Localisation géographique.

\section{HISTORIQUE DES RECHERCHES}

En 1954, durant le décapage du terrain pour la construction d'édifices publiques à Puig d'en Roca près de la ville de Girona, on a découvert une nécropole néolithique où les tombes renfermaient des galets taillés. Ainsi était découvert le complexe à galets taillés d'âge Pléistocène moyen de Puig d'en Roca.

En 1972 l'Association archéologique de Girona a entrepris, sous la direction de J. Canal, la prospection de surface. A partir de ce moment-là on peut distinguer trois périodes de recherche. La première (1972-1977) est consacrée à la prospection de surface ; à la seconde (1977-1979) sont effectués plusieurs sondages dans les sédiments recouvrant les troisième et quatrième terrasses du Ter ; durant la dernière phase (1979-1985) l'Association archéologique de Girona et le Centre de Recherche Paléo-écosociale ont entrepris une fouille systématique du site dit « Puig d'en Roca excavacion» (PREX).

\section{GÉOLOGIE ET GÉOMORPHOLOGIE}

Au cours du Quaternaire le Ter a déposé quatre terrasses au niveau de la ville de Girona (fig. 2). Selon Palli (1976) les différentes terrasses (I à IV) se sont développées à diverses altitudes comme le montre le tableau 1. La proportion de galets quartzeux (d'après Palli) et l'indice d'aplatissement des 
Fïg. 2 - Terrasses quaternaires du Ter à Girona (d'après Palli, 1976).

quartz (Cailleux et Tricart) sont indiqués au tableau 2.

L'industrie du gisement de Puig d'en Roca EX se trouve dans un colluvion à débris de Quartz en contrebas de la quatrième terrasse du Ter (IV) située à $80 \mathrm{~m}$ au-dessus du niveau de la rivière. Ce colluvion est au-dessus d'un lambeau alluvial (terrasse III) à une altitude relative de $45 \mathrm{~m}$, lequel surmonte un encroûtement calcaire renfermant une industrie à galets probablement acheuléenne ; le niveau de $45 \mathrm{~m}$ pourrait donc être rissien.

L'âge de la haute terrasse (IV) nous est indiqué par la composition pétrographique des galets. La terrasse IV, la plus ancienne, ne comporte pas d'éléments basaltiques; par contre la terrasse III en contient une proportion notable (Palli, 1976). L'absence de galets basaltiques dans la terrasse supérieure indique qu'elle s’est déposée antérieurement aux phénomènes volcaniques datés par Donville de 0,11 million d'années dans ce secteur (Donville, 1973).

La position haute de la terrasse IV, son état de démantèlement poussé à l'extrême, sa composition

\begin{tabular}{|l|c|c|}
\hline \multicolumn{1}{|c|}{$\begin{array}{c}\text { Altitude absolue } \\
\text { (niveau de la mer) }\end{array}$} & $\begin{array}{c}\text { Altitude relative } \\
\text { (niveau du Ter) }\end{array}$ \\
\hline Ter & $52 \mathrm{~m}$ & - \\
Terrasse I & $55 \mathrm{~m}$ & $3 \mathrm{~m}$ \\
Terrasse I & $60 \mathrm{~m}$ & $8 \mathrm{~m}$ \\
Terrasse II & $97-100 \mathrm{~m}$ & $45-48 \mathrm{~m}$ \\
Terrasse IV & $130-132 \mathrm{~m}$ & $78-80 \mathrm{~m}$ \\
\hline
\end{tabular}

Tahlean L Altitudes absolue et relative des terrasses

\section{Illustration non autorisée à la diffusion}

Tableau 2 - Proportion de galets quartzeux (Palli) et indice d'aplatissement des quartz. (Cailleux et Tricart). pétrographique, sa position au-dessus d'une terrasse à industrie probablement rissienne nous inciteraient à lui attribuer un âge au moins mindélien.

Le site fouillé par E. Carbonell et R. Mora (PREX) (fig. 3) se présente comme un karst développé sur des bancs de calcaire gréseux tertiaires d'âge éocène. La surface et les interbancs des dalles rocheuses sont corrodés et lissés ; les poches ainsi formées sont remplies par un colluvion rouge sablolimoneux qui présente des passées et des lentilles plus graveleuses.

Le tableau 3 résume la géochronologie que nous proposons pour l'ensemble du site.

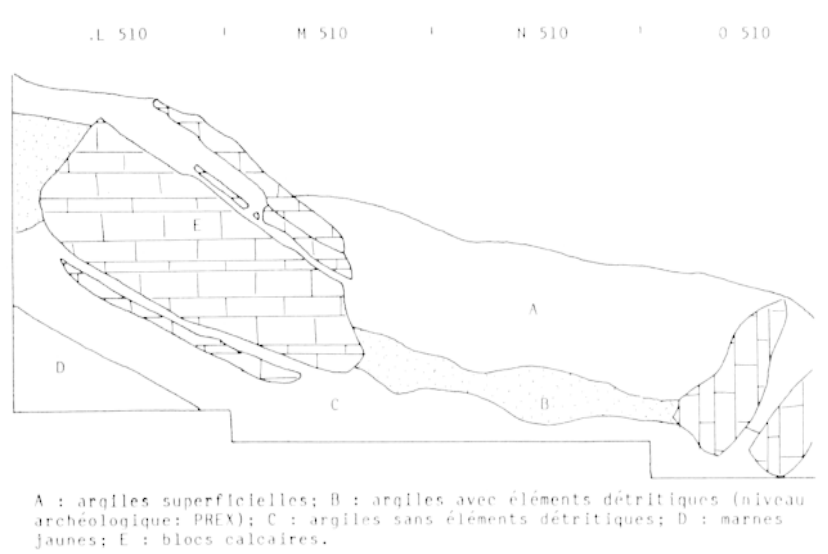

Fig. 3 - PREX : section longitudinale.

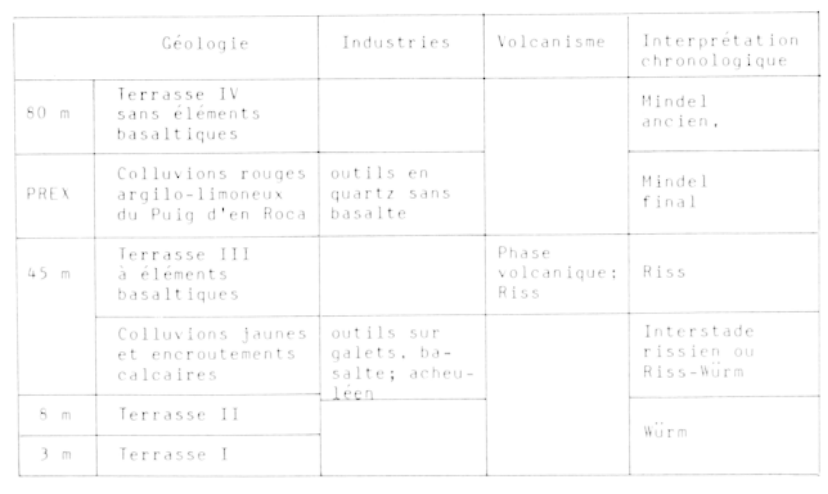

Tableau 3 - Géochronologie du site. 


\section{INDUSTRIE DE PUIG D'EN ROCA EX}

La matière première utilisée et récoltée à Puig d’en Roca EX se compose, pour les bases négatives de première génération (BN1G : galets taillés et « nucléus $"$ ), de $80 \%$ de quartz, $8 \%$ de quartzite et $6 \%$ de porphyre : pour les bases positives (BP : éclats bruts et fragments d'éclats), de $73 \%$ de quartz, $12 \%$ de quartzite et $12 \%$ de porphyre : pour les bases négatives de deuxième génération (BN2G : éclats retouchés), de $85 \%$ de quartz, $9 \%$ de quartzite et $2 \%$ de porphyre.

Au cours des années de fouilles 1979 à 1985 le matériel récolté se répartit selon le tableau 4 . Le classement des objets a été réalisé selon le système logico-analytique (E.C., M.G., R.M., 1983-84). Nous donnons dans cet article un rapide aperçu de notre interprétation du matériel de Puig d'en Roca EX.

\begin{tabular}{|l|rrrr|r|}
\hline Années & 79 & 82 & 84 & 85 & \\
\hline BP & 181 & 205 & 207 & 231 & 824 \\
BHIG & 146 & 116 & 141 & 154 & 557 \\
BH.G & 50 & 83 & 68 & 96 & 297 \\
\hline & 377 & 404 & 416 & 481 & 1678 \\
\hline
\end{tabular}

Tableau 4 - Répartition numérique des objets.

\section{I-Les bases négatives de première génération}

\section{a-vocabulaire général (fig. 4)}

Elles se répartissent en quatre catégories (E.C., M.G., R.M.. 1984) : les unifaces, les bifaces, les trifaces et les multifaces. Parmi ces catégories on peut très schématiquement et d'une façon générale. discerner six manières différentes de tailler la pierre : la taille neutre produisant une arête plus ou moins rectiligne; la taille centripète qui développe des négatifs plus ou moins convergents sur une surface plane ou légèrement bombée ; la taille conique où les enlèvements s'inclinent par rapport au plan théorique contenant l'arête : la taille conique avec arête fortement sinueuse : la taille cylindrique où les enlèvements sont parallèles ou subparallèles et la courbure de l'arête est notable ; enfin la structure conique inverse dont l'angle de taille est ouvert et la courbure de l'arête notable.

La figure 4 donne seulement quelques exemples d'objets simples illustrant diverses possibilités :

U1 : uniface cylindrique : U2 : uniface centripète avec angle de taille proche de 45" ; U3 : deux

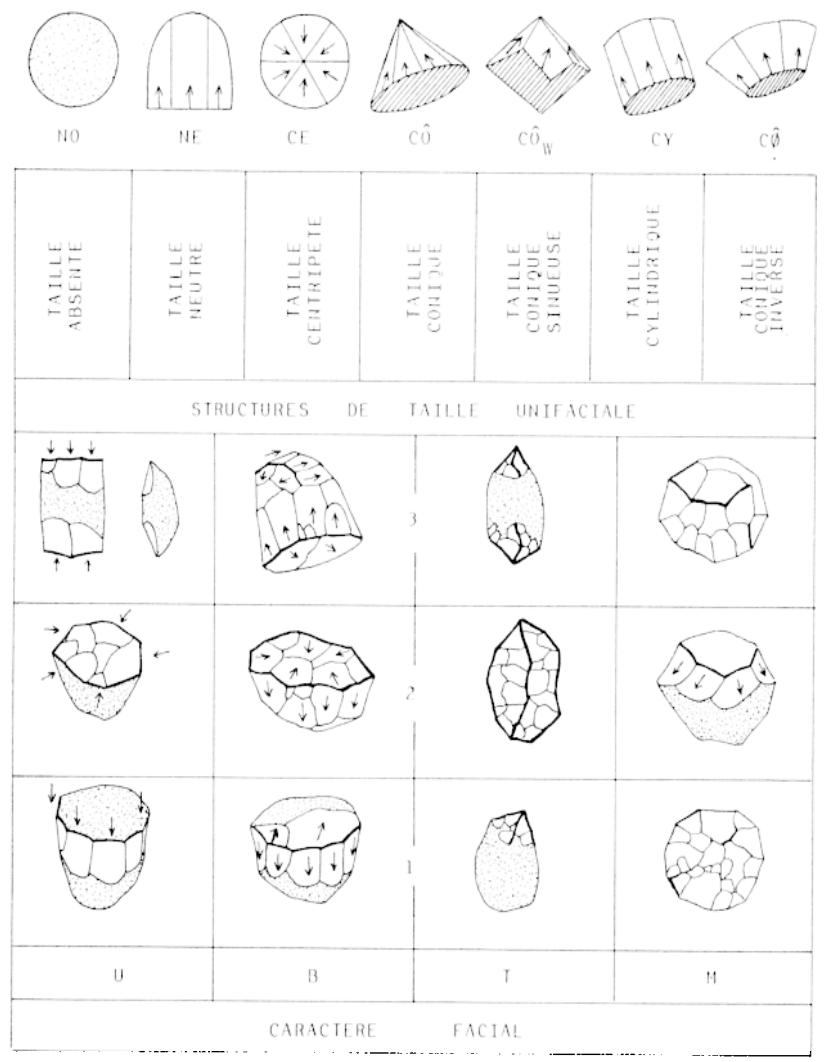

Fig. 4 - Bases négatives de première génération : vocabulaire général.

structures unifaces neutres avec pour chacune un angle de taille voisin de $45^{\circ}$; B1 : biface opposant une structure linéaire à une structure cylindrique ; $\mathrm{B} 2$ : biface opposant une structure centripète à une structure cylindrique ; B3 : deux structures bifaces. Cet objet peut être également considéré comme un triface puisque les arêtes séparent fondamentalement trois faces taillées ; T1 : triface dont les arêtes sont peu développées ; T2 : triface développé ; T3 : deux structures trifaces peu développées. Nous voyons que les trifaces peuvent mêler à des degrés divers les tailles neutre, centripète, conique et cylindrique ; M1 : multiface totalement taillé à multiples structures neutres ; M2 : multiface à taille partielle (aspect exclusivement morphologique). Techniquement, cet objet peut très bien être considéré comme un biface dont une des faces est conique inverse ; M3 : multiface totalement taillé présentant deux ou plusieurs structures bifaces diverses.

\section{$b$ - Puig d'en Roca EX (fig. 5)}

Ce petit vocabulaire simplifié va maintenant nous aider à orienter rapidement nos observations des bases négatives de première génération à Puig d'en Roca EX.

Tout d'abord le caractère facial souligne l'importance des unifaces $(58 \%)$ et des bifaces $(38 \%)$ par rapport aux multifaces $(4 \%)$ et aux trifaces (repré- 


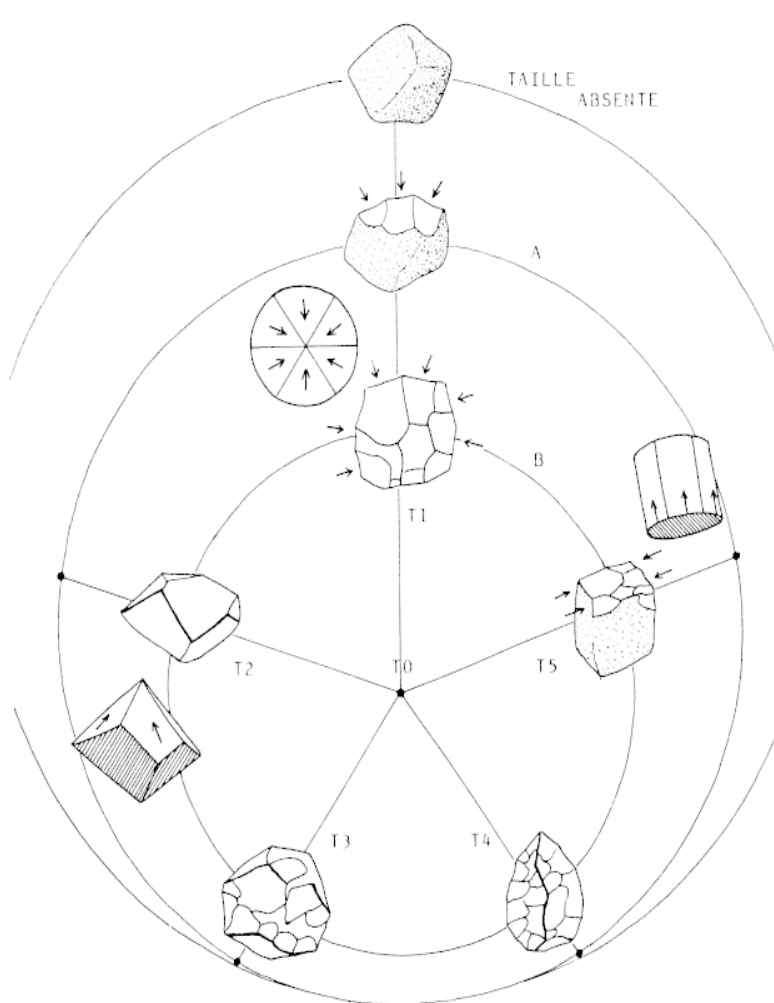

Fig. 5 - Aspect morphotechnique empirique des bases négatives de première génération à Puig d'en Roca EX.

sentés par deux exemplaires). Nous complétons cette analyse quantitative par une orientation empirique destinée à délimiter, d'après la réalité observée, un champ morphotechnique au moyen de quelques thèmes très différenciés (T1 à T5). Ceux-ci se disposent autour du thème cental $(\mathrm{T} 0)$ dont les caractéristiques sont plus ou moins communes avec les thèmes périphériques précités. Il va s'en dire qu'entre tous ces thèmes propres au gisement étudié, il existe un vaste champ de possibilités intermédiaires assurant une bonne cohésion à notre ensemble.

Le thème $T 1$ sous sa forme non développée $(A)$ et développée $(\mathrm{B})$ est très fortement représenté à Puig d'en Roca EX et domine les autres thèmes. Il présente une taille uniface ou biface de type centripète ; naturellement le caractère centripète est croissant lorsque l'on passe de A à B. En outre l'analyse révèle la dominance des unifaces en $A$ et celle des bifaces en B. Soulignons que les bifaces sont constitués pour beaucoup d'une taille centripète opposée à une taille cylindrique ou conique.

T2 est représenté par des objets bifaces dont les enlèvements sont larges et l'arête fortement sinueuse ; la progression du débitage est de type conique sur les deux faces.

T3 est caractéristique des multifaces et T4 des trifaces.

T5 développe une taille cylindrique ; il peut y avoir deux structures opposées et donc deux arêtes comme dans le cas des trifaces.

Beaucoup de pièces se situent, à des degrés divers, entre tous ces thèmes et montrent ainsi de façon évidente la cohésion de cet ensemble industriel du Pléistocène moyen.

Les planches 1 et 2 donnent quelques exemples de BN1G recueillies à Puig d'en Roca EX :

planche $1 ; n^{\circ} 1$ : biface avec structure centripète développée (T1-B), $\mathrm{n}^{\circ} 2$ : uniface centripète développé triangulaire (T1-B), n' 3 : biface très grossièrement biconique avec arête fortement sinueuse (T2), $\mathrm{n}^{\prime \prime} 4$ : uniface centripète à tendance conique, peu développé (T1-A), n" 5 : triface développé (T4), n" 6 : opposition de deux unifaces cylindriques sur la tranche de l'objet (T5) ;

planche $2 ; \mathrm{n}^{\prime \prime} 1$ : caractères de T1-B, T2, T4 et $\mathrm{T} 5$; à partir de cet objet on peut très facilement développer une taille multiface (T3). Nous reconnaissons donc ici le thème central $\mathrm{T} 0 . \mathrm{N}^{\circ} 2$ : proche de $\mathrm{T} 0$ avec toutefois dominance des caractères de $\mathrm{T} 2$ et T5. No 3 : entre T1 et T2 essentiellement. $\mathrm{N}^{\circ} 4$ : entre T1 et T5 essentiellement. $\mathrm{N}^{\circ} 5$ : biface avec structure centripète moyennement développée.
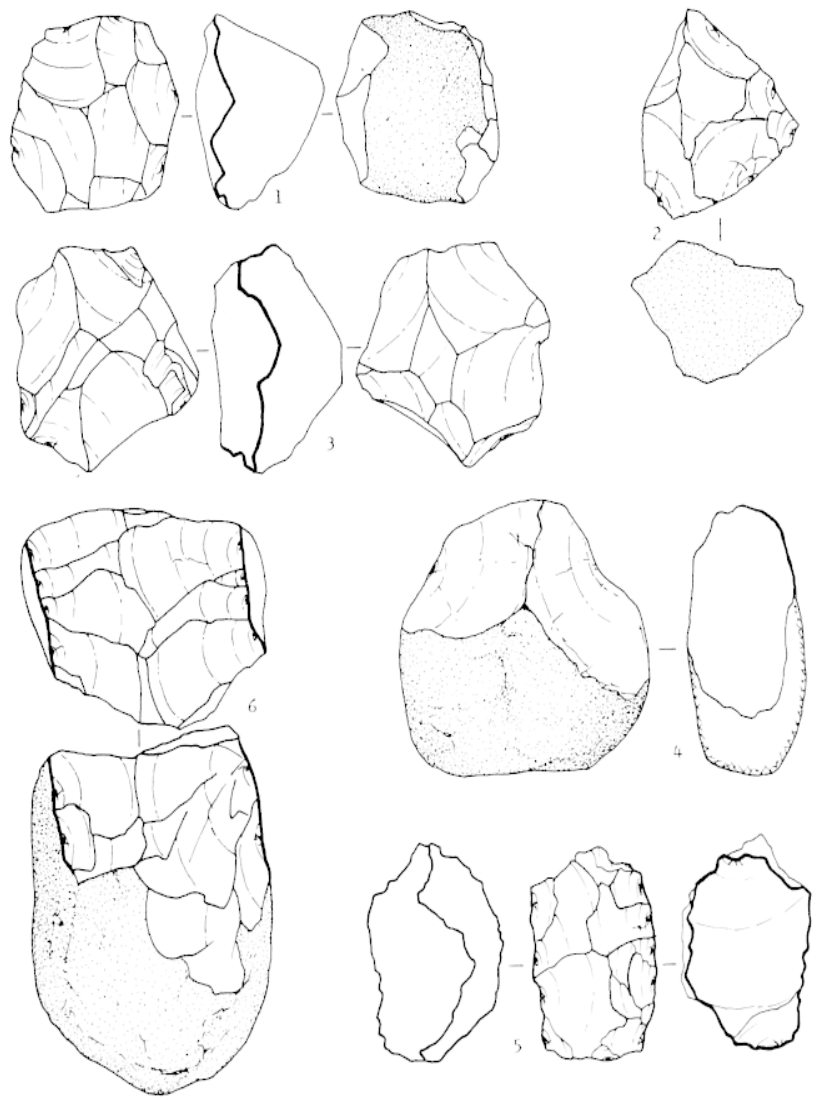

Planche 1 - Bases négatives de première génération à Puig d'en Roca EX. 


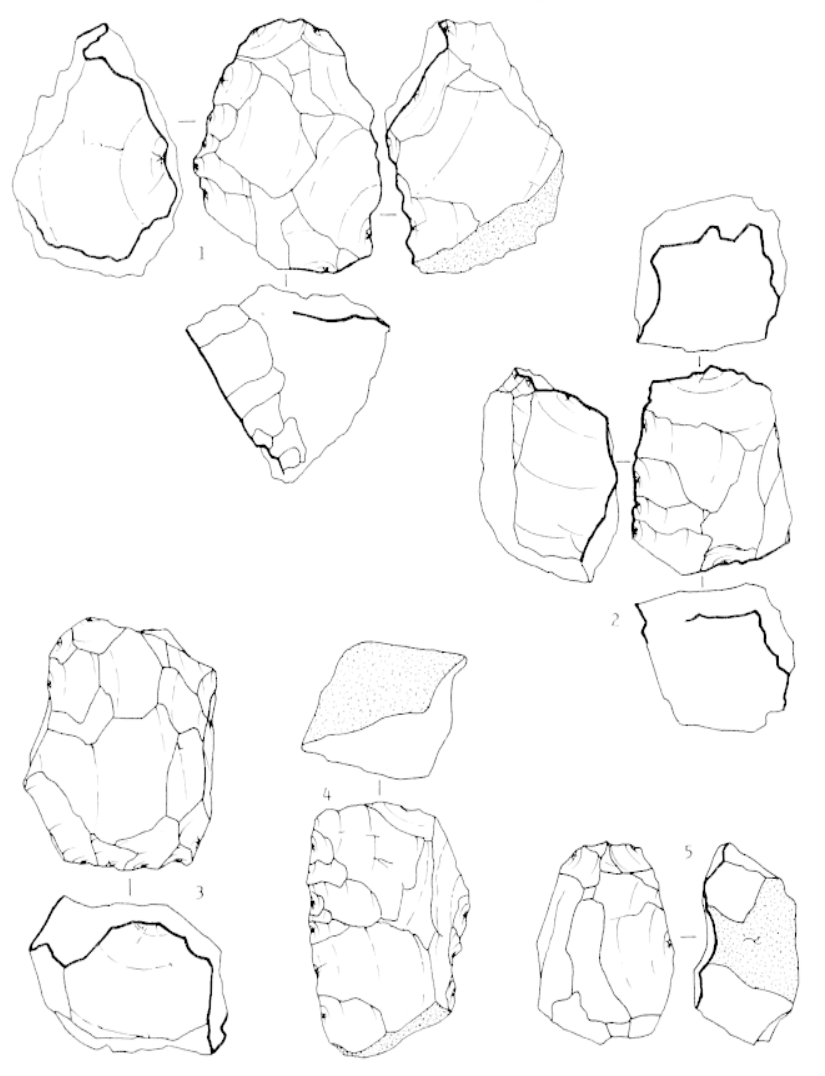

Planche 2 - Bases négatives de première génération à Puig d'en Roca EX.

\section{2 - Les bases positives (planche 3 )}

La face ventrale des bases positives présente une morphologie convexe dans leur section sagittale. Les talons sont en général non corticaux, à plan de frappe plus ou moins étendu, unifacetté, rectiligne ou convexe. La face dorsale est très souvent non corticale ou semi-corticale.

3 - Les bases négatives de deuxième génération (planche 3)

Elles présentent des talons souvent non corticaux avec plan de frappe étendu, unifacetté et rectiligne. Les retouches simples $(43 \%)$ et abruptes $(38 \%)$ dominent les retouches plates $(18 \%)$; il y a quelques burins. La profondeur des retouches est à $80 \%$ marginale ou très marginale. La forme obtenue est pour $44 \%$ convexe, concave pour $22 \%$ et rectiligne pour $21 \%$; les uniangulaires représentent $12 \%$.

En résumé l'industrie de Puig d'en Roca EX est caractérisée d'une part par l'exploitation essentiellement centripète des matières premières pour l'obtention d'éclats courts, et d'autre part par le peu de modification des bases positives. Il n'existe pas de morphotypes caractéristiques dans l'ensemble des bases négatives de deuxième génération. La mau-

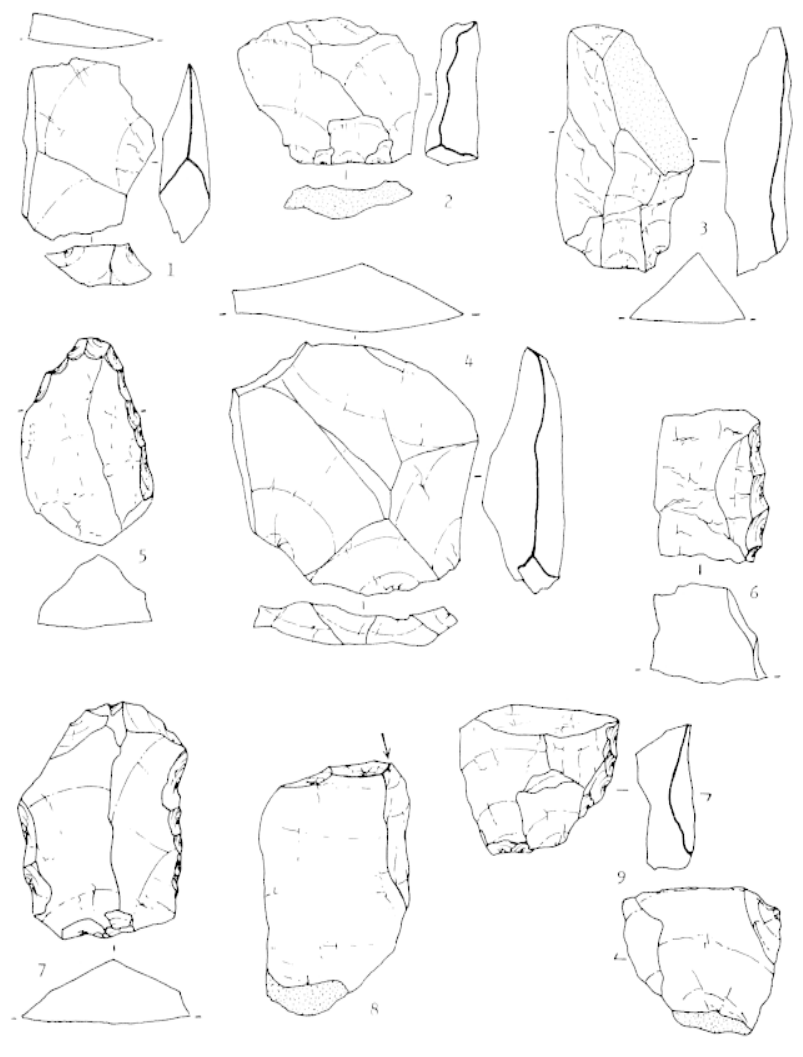

Planche 3 - Bases positives $\left(n^{\text {os }} 1\right.$ à 4 ) et bases négatives de deuxième génération ( ${ }^{\circ 5} 5$ à 9 ) à Puig d'en Roca EX.

vaise qualité des matières premières (quartz filonien) et l'utilisation massive des fragments limitent dans la majorité des cas le développement des formes bien différenciées.

CONTEXTE GÉNÉRAL

Les terrasses étagées sur la rive gauche de la Têt, entre Millas et Perpignan ont fourni des industries mindéliennes (Collina-Girard J., 1975) (fig. 1). La relative proximité géographique des deux bassins versants autorise des comparaisons. L'indication des altitudes relatives (tableau 5) n'écarte pas l'hypothèse d'un âge au moins mindélien pour la terrasse IV de Puig d'en Roca. Sa position altimétrique relative est à rapprocher en effet de celle des niveaux les plus anciens du Roussillon: Mas Ferréol et Cabestany datés du Villafranchien (Günz).

La terrasse III de Puig d'en Roca occupe une position analogue à celle de la Butte du Four dans la région de Millas.

L'âge mindélien de l'industrie de Puig d'en Roca EX est renforcé par sa relative proximité mor- 


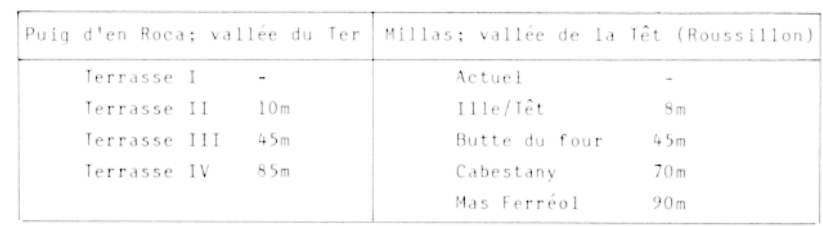

Tableau 5 - Altitudes relatives des terrasses du Ter et du Roussillon (Têt).

photechnique avec l’industrie de La Labanère (terrasse de la Têt) (E.C.. M.G.. R.M. 1982) rapportée au Mindel (Collina-Girard. 1975).

E. Carbonfit. R. Mora et R. Sala Museu d'historia de la ciutat La Força. 27 $17(0) 1$ Gerone Espagne

J. (OI.IINA-(iRARI) Institut du Quaternaire

Bâtiment de Géologic Avenue des Facultés 33405 Talence Cedex

M. GLill Bal'I) 58. rue Gilbert 16100 Cognac
Carbonila. E. Glifibaldo M. et Mora R. (1982) - Application de la méthode dialectique à la construction d'un svstème analỵtique pour l'étude des matériaux du Paléolithique inférieur. Dialcktiké. Cahiers de typologie analytique, Pau. pp. 7 23.

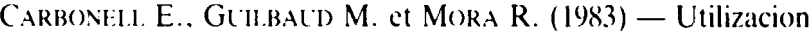
de la logica analitica para el estudio de tecno-complejos a cantos tallados. Cahier noir. Girona. éd. G.I.P.E.S.. n"1.pp. 1-6t.

Carbonili. E.. Gill.balis) M. el Mora R. (1984) - Amplification du svsteme analytique ave la classification des technocomplexes à galets taillés. Bull. S.P.F.. 1. 81, n"7. pp. 203-206, 1 fig.

Coll.NAA-GIRARI) J. (1975) - Les industries archaïques sur galets des terrasses quaternaires de la plaine du Roussillon (P.-O.. France). Travaux du laboratoire de Paléontologie humaine et de Préhistoire de l'Université de Provence, Marseille. mémoire n"1.

Donvil.1: B. (1973) - Géologie néogène et âge des ćruptions, volcaniques de la Catalogne orientale. Thèse de doctorat. Toulousc. Languedoc.

G(II.BA(1) M. (1987) - Le débitage comme expression d'une réalité psychique. 1" Réunion internationale sur les systèmes d analyse en archéologic. C.R.P.E.S., Girona.

PAl.1. L. (1976) - Morfolitologia de las terrazas del Ter en Girona. Anales de la Seccion de Ciencas del Colegio universitario de (jirona, Ano I, n" I. Junio 1976 